Bangladesh J. Plant Taxon. 24(2): 165-171, 2017 (December)

(C) 2017 Bangladesh Association of Plant Taxonomists

\title{
NEW ANGIOSPERMIC TAXA FOR THE FLORA OF BANGLADESH
}

\author{
M. Oliur Rahman ${ }^{1}$ and Md. Abul Hassan \\ Department of Botany, University of Dhaka, Dhaka 1000, Bangladesh
}

Keywords: New records; New species; Angiosperm; Flora of British India; Bengal Plants.

\begin{abstract}
This paper presents addition of 89 taxa under 64 genera distributed in 32 families for the flora of Bangladesh which are not included in the monumental works Flora of British India, Bengal Plants and Encyclopedia of Flora and Fauna of Bangladesh. Updated nomenclature, family name, references to the work and the precise localities have been furnished under each taxon.
\end{abstract}

\section{Introduction}

Bangladesh is a reservoir of plant resources comprising 3,611 angiosperm taxa occurring in the country (Ahmed et al., 2007-2009). Despite several floristic studies were carried out over last four decades after the emergence of Bangladesh the botanical expedition throughout the country is yet to be completed. Hooker (1872-1897) and Prain (1903) made significant contribution on floristic studies in the Indian subcontinent and reported many species from the territory of present Bangladesh. Some regional flora of Bangladesh have also been produced by several workers (Heinig, 1925; Cowan, 1926; Raizada, 1941; Datta and Mitra, 1953; Sinclair, 1956).

In the recent past several authors paid attention to explore the flora of Bangladesh and recorded many taxa as new for the country. Mia and Khan (1995) published the first list of angiospermic taxa, not included in the Flora of British India (Hooker, 1872-1897) and Bengal Plants (Prain, 1903), adding a total of 325 species as recorded by several workers up to that time for Bangladesh. Rahman (2004a, b) added 138 angiosperm taxa to our knowledge on the flora of Bangladesh. Recently, Ahmed et al. (2007-2009) documented all species available in Bangladesh in the Encyclopedia of Flora and Fauna of Bangladesh adding several new angiosperm records for the country. After the momentous contribution made by Ahmed et al. (2007-2009) a large number of taxa have been added further, either as new records or as new taxa for the flora of Bangladesh. Therefore, the present study aimed at preparing a comprehensive checklist of all the new additions published either as new species or new records for the angiosperm flora of Bangladesh since Ahmed et al. (2007-2009).

\section{Materials and Methods}

This study was based on published works on new angiosperm records and new species for Bangladesh. All the relevant published papers and literature (Hooker, 1872-1897; Prain, 1903; Mia and Khan, 1995; Rahman, 2004a, b; Ahmed et al., 2007-2009) have been consulted in order to update and finalize the newly reported taxa of Bangladesh. The new taxa are arranged in an alphabetical order and presented along with their updated nomenclature, family name, reference to the work and the precise locality.

\section{Results}

A total of 89 taxa under 64 genera belonging to 32 families have been added to the previous lists as new records or new species for Bangladesh. Despite some other new records or taxa

\footnotetext{
${ }^{1}$ Corresponding author. Email: prof.oliurrahman@gmail.com
} 
identified for the flora of Bangladesh they are yet to be published and are not included in this paper. The taxa recorded here as new for the country are presented below.

1. Alchornea mollis Benth. ex Mull.-Arg. (Euphorbiaceae). Uddin et al. (2015d). p. 89. Moulvi Bazar: Srimangal, Harinchara.

2. Allophylus samarensis Merr. (Sapindaceae). Uddin et al. (2015c). p. 78. Moulvi Bazar: Madahbkundo Eco-park.

3. Amorphophallus excentricus Helt (Araceae). Ara and Hassan (2012). p. 18. Moulvi Bazar: Madhabkundo forest.

4. Amorphophallus krausei Engl. (Araceae). Ara and Hassan (2012). p. 18. Moulvi Bazar: Adampur beat, Kawargola forest; Lawachara reserve forest.

5. Ancistrocladus tectorius (Lour.) Merr. (Ancistrocladaceae). Uddin et al. (2015d). p. 90. Moulvi Bazar: Kamalganj, Lawachara National Park; Madhabkundo Eco-park.

6. Argostemma sarmentosumWall. (Rubiaceae). Das and Rahman (2010). p. 216. Rangamati: Shubalong.

7. Aristolochia coadunata Back. (Aristolochiaceae). Uddin et al. (2015b). p. 69. Rangamati: Pharua reserve forest, Bilaichari.

8. Aspidopteris tomentosa (Bl.) Juss. (Malpighiaceae). Uddin and Hassan (2015). p. 35. Rangamati: Kaptai, Rampahar.

9. Atalantia kwangtungensis Merr. (Rutaceae). Uddin et al. (2015c). p. 79. Moulvi Bazar: Madahbkundo Eco-park.

10. Begonia rubella Buch.-Ham. ex D. Don (Begoniaceae). Uddin and Hassan (2015). p. 36. Rangamati: Kaptai, Sitapahar.

11. Beilschmiedia sikkimensis King ex Hook. f. (Lauraceae). Uddin and Hassan (2015). p. 38. Rangamati: Kaptai, Rampahar.

12. Boehmeria aspera Wedd. (Urticaceae). Uddin et al. (2015a). p. 2. Rangamati: Pharua reserve forest, Bilaichari.

13. Boehmeria clidemioides Miq. (Urticaceae). Uddin et al. (2015a). p. 3. East Bengal (CAL).

14. Boehmeria hamiltoniana Wedd. (Urticaceae). Uddin et al. (2015a). p. 5. East Bengal (CAL).

15. Boehmeria manipurensis Friis \& Wilmot-Dear (Urticaceae). Uddin et al. (2015a). p. 6. Rangamati: Sapchari; Khagrachari: Gomoti, Panchari.

16. Boeica filiformis Clarke (Gesneriaceae). Uddin et al. (2015c). p. 81. Moulvi Bazar: Madahbkundo Eco-park.

17. Brachycorythis obcordata (Lindl.) Summerh (Orchidaceae). Hoque and Huda (2008). p. 193. Bandarban: Chimbuk.

18. Catunaregam longispina (Link) Tirveng. (Rubiaceae). Das et al. (2013). p. 258. Gazipur: Chandra forest; Habigonj: Chanbari, Rema-Kalenga Wildlife Sanctuary; Sherpur: Gajni forest.

19. Chlorophytum nepalense (Lindley) Baker (Liliaceae). Afroz et al. (2008). p. 193. Sherpur: Runctia sal forest.

20. Colocasia virosa Kunth (Araceae). Ara and Hassan (2012). p. 19. Moulvi Bazar: Muraichara beat, Ichachara forest.

21. Colubrina javanica Miq. (Rhamnaceae). Rahman et al. (2014). p. 199. Bagerhat: Sundarbans east forest division, Katka, near forest station.

22. Cryptocarya calderi M. Gangop. (Lauraceae). Uddin and Hassan (2015). p. 39. Cox’s Bazar: Dulahazara safari park; Rangamati: Kaptai, Sitapahar. 
23. Cucumis hystrix Chakravarty (Cucurbitaceae). Uddin et al. (2012). p. 205. Rangamati: Bilaichari, Pharua reserve forest.

24. Cuphea carthagenensis (Jacq.) J.F. Macbr. (Lythraceae). Hossain et al. (2015). p. 115. Sylhet: Lacctura; Shahjalal University of Science and Technology campus; Jaflong.

25. Curcuma bakerii Rahman \& Yususf (Zingiberaceae). Rahman (2012). p. 121. Tangail: Madhupur sal forest.

26. Curcuma hookerii Rahman \& Yusuf (Zingiberaceae). Rahman (2012). p. 123. Chittagong: Barabkundu.

27. Curcuma roxburghii Rahman et Yusuf (Zingiberaceae). Rahman and Yusuf (2012). p. 80. Rangamati: Rangapani.

28. Curcuma wallichii Rahman et Yusuf (Zingiberaceae). Rahman and Yusuf (2012). p. 82. Maulvi Bazar: Srimongal, Lawachara rain forest.

29. Curcuma wilcockii Rahman et Yusuf (Zingiberaceae). Rahman and Yusuf (2012). p. 83. Tangail: Madhupur sal forest, Rasulpur; Sylhet: Tamabil.

30. Dianella ensifolia (L.) DC. (Liliaceae). Uddin and Hassan (2009). p. 181. Rangamati: Kaptai, Rampahar.

31. Diospyros albiflora Alston (Ebenaceae). Sultana et al. (2010). p. 249. Patuakhali: Mirjagong.

32. Egeria densa Planchón (Hydrocharitaceae). Alfasane et al. (2010). p. 210. Bandarban: Bogakain lake.

33. Elatostema dissectum Wedd. (Urticaceae). Uddin et al. (2015a). p. 7. Chittagong Hill Tracts: Mynimukh (CAL).

34. Elatostema ellipticum Wedd. (Urticaceae). Uddin et al. (2015a). p. 8. East Bengal (K).

35. Elatostema griffithii Hook. f. (Urticaceae). Uddin et al. (2015a). p. 9. East Bengal (K).

36. Elatostema obtusum Wedd. (Urticaceae). Uddin et al. (2015a). p. 9. East Bengal (CAL).

37. Elatostema procridioides Wedd. (Urticaceae). Uddin et al. (2015a). p. 11. East Bengal (CAL).

38. Elatostema subincisum Wedd. (Urticaceae). Uddin et al. (2015a). p. 12. Chittagong (CAL).

39. Embelia parviflora Wall. ex A. DC. (Myrsinaceae). Uddin et al. (2015e). p. 96. Moulvi Bazar: Juri forest range, Lathitilla forest beat.

40. Galium pusillosetosum Hara (Rubiaceae). Das et al. (2013). p. 259. Chittagong: Baluchara.

41. Guazuma ulmifolia Lam. (Sterculiaceae). Mia et al. (2011). p. 154. Noakhali.

42. Gynura nepalensis DC. (Asteraceae). Afroz et al. (2014). p. 101. Dhaka: Dhaka University Botanical Garden; Netrakona: Kendua.

43. Helicteres viscida Bl. (Sterculiaceae). Mia et al. (2011). p. 154. Chittagong: Jaldi Range, Bilaichori.

44. Hemiorchis rhodorrhachis Schum. (Zingiberaceae). Srivastava and Ghoshal (2005). p. 59. Chittagong Hill Tracts: Barkal.

45. Hydrocotyle verticillata Thunb. (Apiaceae). Khatun et al. (2010). p. 105. Dhaka: Azimpur.

46. Ilex glomerata King (Aquifoliaceae). Uddin et al. (2015d). p. 91. Moulvi Bazar: Lawachara National Park.

47. Illigera khasiana C.B. Clarke (Hernandiaceae). Uddin et al. (2015b). p. 70. Rangamati: Pharua reserve forest, Bilaichari.

48. Laportea bulbifera (Siebold \& Zuccarini) Wedd. (Urticaceae). Uddin et al. (2015a). p. 13. East Bengal (CAL). 
49. Lindera neesiana (Wall. ex Nees) Kurz (Lauraceae). Ara and Khan (2015). p. 28. East Bengal (CAL).

50. Litsea khasyana Meissn. (Lauraceae). Ara and Khan (2015). p. 29. East Bengal (CAL, K).

51. Litsea umbellata (Lour.) Merr. (Lauraceae). Ara and Khan (2015). p. 29. East Bengal (CAL).

52. Maytenus hookeri Loes. (Celastraceae). Uddin and Hassan (2015). p. 40. Rangamati: Kaptai, Rampahar.

53. Mitrephora grandiflora Beddome (Annonaceae). Uddin and Hassan (2015). p. 42. Rangamati: Kaptai, Sitapahar.

54. Mussaenda incana Wall. ex Roxb. (Rubiaceae). Das and Rahman (2010). p. 217. Chittagong: Sitakunda, Eco-Park area; Moulvi Bazar: Srimongal, Bhanugach road.

55. Mussaenda keenani Hook. f. (Rubiaceae). Das et al. (2012). p. 22. Chittagong: Korerhat, Koila, Guichari; Rangamati: Kaptai, Rampahar, Madhabchari; Moulvi Bazar: Srimongal, Lawachora forest.

56. Mycetia listeri Deb. (Rubiaceae). Das et al. (2012). p. 23. Chittagong: Jamaichari; Rangamati: Kaptai, Rampahar.

57. Mycetia malayana (G. Don) Craib. (Rubiaceae). Uddin and Rahman (2015). p. 104. Chittagong: Dohazari, Lalutia; Moulvi Bazar: Madhabkundo Eco-park; Rangamati: Kaptai, Sitapahar west.

58. Mycetia mukerjiana Deb \& Dutta (Rubiaceae). Das and Rahman (2010). p. 218. Rangamati: Kaptai, Sitapahar Wildlife Sanctuary.

59. Mycetia sinensis (Hemsley) Craib (Rubiaceae). Uddin and Rahman (2015). p. 107. Moulvi Bazar: Kamalganj, Lawachara National Park.

60. Mycetia stipulata (Hook. f.) O. Kuntze subsp. macrostachya (Hook. f.) Deb (Rubiaceae). Uddin and Rahman (2015). p. 108. East Bengal (K).

61. Neodistemon indicum (Wedd.) Babu \& Henry (Urticaceae). Uddin et al. (2015a). p. 14. Moulvi Bazar: Madhabkundo Eco-park; Rangamati: Kaptai, Rampahar.

62. Ophiorrhiza eriantha Wight (Rubiaceae). Das et al. (2013). p. 260. Khagrachari: Shilchari, Alu tila.

63. Ophiorrhiza fasciculata D. Don (Rubiaceae). Das et al. (2012). p. 24. Chittagong: Dhopachari, Gondamara; Rangamati: Kutukchari, Chegaiya-chari.

64. Oxyceros rugulosus (Thwaites) Tirveng. (Rubiaceae). Das and Rahman (2010). p. 219. Chittagong: Hazarikhil; Cox’s Bazar: Teknaf; Rangamati: Pablakhali; Sylhet: Jafflong.

65. Pellionia heteroloba Wedd. (Urticaceae). Uddin et al. (2015a). p. 16. East Bengal (CAL, K).

66. Pellionia heyneana Wedd. (Urticaceae). Uddin et al. (2015a). p. 17. Rangamati: Pharua reserve forest, Bilaichari.

67. Pellionia repens (Lour.) Merr. (Urticaceae). Uddin et al. (2015a). p. 18. Dhaka: Balda garden.

68. Phenax mexicanus Wedd. (Urticaceae). Uddin et al. (2015a). p. 19. Rangamati: Kaptai, Karnaphuli sadar beat; Pharua reserve forest, Bilaichari.

69. Phyllanthus columnaris Muell.-Arg. (Euphorbiaceae). Uddin and Hassan (2015). p. 43. Rangamati: Kaptai, Rampahar.

70. Pilea anisophylla Wedd. (Urticaceae). Uddin et al. (2015a). p. 20. East Bengal (K).

71. Pilea bracteosa Wedd. (Urticaceae). Uddin et al. (2015a). p. 22. East Bengal (K). 
72. Pilea insolens Wedd. (Urticaceae). Uddin et al. (2015a). p. 23. East Bengal (K, CAL).

73. Pollia thyrsiflora (Bl.) Endley ex Hassk. (Commelinaceae). Uddin and Hassan (2015). p. 44. Rangamati: Kaptai, Sitapahar, Jamaichara.

74. Psychotria stipulacea Wall. (Rubiaceae). Das et al. (2012). p. 25. Rangamati: Kaptai, Sitapahar.

75. Psydrax umbellata (Wight) Bridson (Rubiaceae). Das et al. (2012). p. 26. Chittagong: Bomariaghona; Sylhet: Tamabil.

76. Pulicaria vulgaris Gaertn. (Asteraceae). Rahman et al. (2011). p. 205. Patuakhali: Galachipa.

77. Pyrenaria diospyricarpa Kurz (Theaceae). Uddin et al. (2015b). p. 71. Rangamati: Pharua reserve forest, Bilaichari.

78. Sarcopyramis napalensis Wall. (Melastomataceae). Uddin et al. (2015e). p. 98. Moulvi Bazar: Juri forest range, Lathitilla forest beat.

79. Sida spinosa L. (Malvaceae). Shetu et al. (2015). p. 111. Dhaka: Near Kafrul thana, Mirpur; Khulna: Khulna University campus.

80. Spermacoce exilis (Williams) Adams ex Burger et Taylor (Rubiaceae). Das et al. (2013). p. 261. Chittagong: Kumira, Dardorir chara; Cox’s Bazar: Himchari, Bhangamura; Laksmipur: Ramgonj; Moulvi Bazar: Srimongal, Tea resort; Sylhet: Tibbi College campus.

81. Staurogyne simonsii (Anders.) O. Kuntze (Acanthaceae). Uddin et al. (2015e). p. 99. Moulvi Bazar: Juri forest range, Lathitilla forest beat.

82. Sterculia urens Roxb. (Sterculiaceae). Mia et al. (2011). p. 155. Chittagong.

83. Steudnera gagei Krause (Araceae). Ara and Hassan (2012). p. 20. Moulvi Bazar:Adampur beat, Gangpali.

84. Tarenna helferi (Kurz) N.P. Balakr. (Rubiaceae). Das and Rahman (2010). p. 220. Rangamati: Betbunia, Mahajan para; Moulvi Bazar: Srimongal, Lawachara forest.

85. Tarenna stellulata (Hook. f.) Ridl. (Rubiaceae). Das et al. (2013). p. 262. Chittagong: Sitakunda, Chandranath hill.

86. Trigonostemon viridissimus (Kurz) Airy Shaw (Euphorbiaceae). Uddin et al. (2015c). p. 82. Moulvi Bazar: Madahbkundo Eco-park.

87. Vanilla havilandii Rolfe (Orchidaceae). Uddin et al. (2015b). p. 73. Rangamati: Pharua reserve forest, Bilaichari, Monlovi chara.

88. Xanthosoma undipes (K. Koch) K. Kock (Araceae). Ara and Hassan (2012). p. 22. Gazipur: Kamesshor village.

89. Zingiber salarkhanii (Zingiberaceae). Rahman and Yusuf (2013). p. 240. Chittagong: Sitakundu, Chandranath hill; Khagrachari: Teen Tila, Marissa road, Moulvi Bazar: Srimongal, Lawachara reserve forest.

\section{References}

Afroz, S., Tutul, E., Uddin, M.Z. and Hassan, M.A. 2008. Chlorophytum nepalense (Lindley) Baker (Liliaceae) - A new angiospermic record for Bangladesh. Bangladesh J. Bot. 37(2): 193-194.

Afroz, S., Uddin, M.Z. and Hassan, M.A. 2014. Gynura nepalensis DC. (Asteraceae) - A new angiosperm record for Bangladesh. Bangladesh J. Plant Taxon. 21(1): 101-104.

Ahmed, Z.U., Begum, Z.N.T., Hassan, M.A., Khondker, M., Kabir, S.M.H., Ahmad, M., Ahmed, A.T.A., Rahman, A.K.A. and Haque, E.U. (Eds) 2007-2009. Encyclopedia of Flora and Fauna of Bangladesh, Vols. 6-12. Asiatic Society of Bangladesh, Dhaka. 
Alfasane, M.A.,Khondker, M.,Islam, M.S. and Bhuiyan, M.A.H. 2010. Egeria densa Planchón (Hydrocharitaceae) - A new angiospermic record for Bangladesh. Bangladesh J. Plant Taxon. 17(2): 209-213.

Ara, H. and Hassan, M.A. 2012. Five new records of aroids for Bangladesh. Bangladesh J. Plant Taxon. 19(1): 17-23.

Ara, H. and Khan, B. 2015. Three new records of Lauraceae from Bangladesh. Bull. Bangladesh National Herb. 4: 27-32.

Cowan, J.M. 1926. The flora of Chakaria Sundarbans. Rec. Bot. Surv. Ind. 11: 197-225.

Das, S.C. and Rahman, M.A. 2010. Notes on the Rubiaceae. 3. Five new records for Bangladesh. Bangladesh J. Bot. 39(2): 215-222.

Das, S.C., Dev, P.K. and Rahman, M.A. 2012. Notes on the Rubiaceae - 4: Five new records for Bangladesh. Bangladesh J. Bot. 41(1): 21-28.

Das, S.C., Dev, P.K. and Rahman, M.A. 2013. Notes on the Rubiaceae - 5: Five new records for Bangladesh. Bangladesh J. Bot. 42(2): 257-264.

Datta, R.B. and Mitra, J.N. 1953. Common Plants in and around Dacca city. Bull. Bot. Soc. Beng. 7(1\&2): 1110.

Heinig, R.L. 1925. List of the Plants of Chittagong Collectorate and Hill Tracts. Darjeeling.

Hooker, J.D. 1872-1897. The Flora of British India. Vols. 1-7. L. Reeve \& Co. Ltd., England.

Hoque, M.M. and Huda, M.K. 2008. Brachycorythis obcordata (Lindl.) Summerh. (Orchidaceae) - A new angiospermic record for Bangladesh. Bangladesh J. Bot. 37(2): 199-201.

Hossain, G.M., Khan, M.S.A., Rahman, M.S., Haque, A.K.M.K. and Rahim, M.A. 2015. Cuphea carthagenensis (Jacq.) J.F. Macbr. (Lythraceae) - A new angiosperm record for Bangladesh. Bull. Bangladesh National Herb. 4: 115-117.

Khatun, B.M., Rahman, M.O. and Sultana, S.S. 2010. Hydrocotyle verticillata Thunb. (Apiaceae) - A new angiospermic record for Bangladesh. Bangladesh J. Plant Taxon. 17(1): 105-108.

Mia, M.K. and Khan, B. 1995. First list of angiospermic taxa of Bangladesh not included in Hooker's Flora of British India and Prain's Bengal Plants. Bangladesh J. Plant Taxon. 2(1\&2): 25-45.

Mia, M.M.K., Rahman, M.O., Hassan, M.A. and Huq, A.M. 2011. Three new records of Sterculiaceae for Bangladesh. Bangladesh J. Plant Taxon. 18(2): 153-157.

Prain, D. 1903. Bengal Plants. Vols. 1\&2. (Reprint edition 1963). Botanical Survey of India, Calcutta.

Rahman, M.A. 2012. Discovery of new species from Bangladesh. Plantae Discoverie 1: 1-34.

Rahman, M.A. and Yusuf, M. 2012. Three new species of Curcuma L. (Zingiberaceae) from Bangladesh. Bangladesh J. Plant Taxon. 19(1): 79-84.

Rahman, M.A. and Yusuf, M. 2013. Zingiber salarkhanii (Zingiberaceae) - A new species from Bangladesh. Bangladesh J. Plant Taxon. 20(2): 239-242.

Rahman, M.O. 2004a. Second list of angiospermic taxa not included in Hooker's Flora of British India and Prain’s Bengal Plants - Series I. Bangladesh J. Plant Taxon. 11(1): 77-82.

Rahman, M.O. 2004b. Second list of angiospermic taxa not included in Hooker's Flora of British India and Prain’s Bengal Plants - Series II. Bangladesh J. Plant Taxon. 11(2): 49-56.

Rahman, M.O., Sultana, M., Begum, M. and Hassan, M.A. 2011. Pulicaria vulgaris Gaertn. (Asteraceae) -A new species record for Bangladesh. Bangladesh J. Plant Taxon. 18(2): 205-208.

Rahman, M.S., Hossain, G.M., Khan, S.A. and Uddin, S.N. 2014. Colubrina javanica Miq. (Rhamnaceae) A new angiosperm record for Bangladesh. Bangladesh J. Plant Taxon. 21(2): 199-202.

Raizada, M.B. 1941. On the Flora of Chittagong. Indian Forester 67(5): 245-254.

Shetu, S.S., Khan, M.S.A. and Uddin, S.N. 2015. Sida spinosa L. (Malvaceae) - A new angiosperm species record for Bangladesh. Bull. Bangladesh National Herb. 4: 111-113.

Sinclair, J. 1956. Flora of Cox's Bazar, East Pakistan. Bull. Bot. Soc. Beng. 9(2): 92-94.

Srivastava, S.C. and Ghoshal, P.P. 2005. Hemiorchis rhodorrhachis Schum. (Zingiberaceae) - A new record for Bangladesh. Bangladesh J. Plant Taxon. 12(1): 59-61. 
Sultana, M., Rahman, M.O., Begum, M. and Hassan, M.A. 2010. Diospyros albiflora Alston (Ebenaceae) - A new angiospermic record for Bangladesh. Bangladesh J. Bot. 39(2): 249-251.

Uddin, S.N and Hassan, M.A. 2009. Dianella ensifolia (L.) DC. (Liliaceae) - A new angiospermic record for Bangladesh. Bangladesh J. Plant Taxon. 16(2): 181-184.

Uddin, S.N., Khan, B. and Mirza, M.M. 2012. Cucumis hystrix Chakrav. (Cucurbitaceae) - A new angiospermic record for Bangladesh. Bangladesh J. Plant Taxon. 19(2): 205-207.

Uddin, S.N. and Hassan, M.A. 2015. Discovery of eight angiosperm new records for Bangladesh from Rampahar and Sitapahar reserve forest under Rangamati district. Bull. Bangladesh National Herb. 4: 33-49.

Uddin, S.N. and Rahman, N. 2015. Notes on occurrence of the genus Mycetia Reinwardt (Rubiaceae) in Bangladesh. Bull. Bangladesh National Herb. 4: 103-110.

Uddin, S.N., Khan, B. and Hassan, M.A. 2015a. Nineteen new records of Urticaceae from Bangladesh. Bull. Bangladesh National Herb. 4: 1-25.

Uddin, S.N., Khan, B. and Mirza, M.M. 2015b. Discovery of four angiospermic new records for Bangladesh from Pharua Reserve forest under Rangamati district. Bull. Bangladesh National Herb. 4: 67-76.

Uddin, S.N., Khan, B. and Khokan, M.E.H. 2015c. Discovery of four angiosperm new records for Bangladesh from Madhabkundo Eco-park under Moulvi Bazar district. Bull. Bangladesh National Herb. 4: 77-85.

Uddin, S.N., Khokan, M.E.H. and Khan, B. 2015d. Discovery of three angiosperm new records for Bangladesh from Lawachara National Park under Moulvi Bazar district. Bull. Bangladesh National Herb. 4: 87-94.

Uddin, S.N., Khokan, M.E.H., Khan, B. and Islam, K.K. 2015e. Discovery of three new angiosperm records for Bangladesh from Juri forest range-1 under Moulvi Bazar district. Bull. Bangladesh National Herb. 4: 95-102. 